\title{
Use of Coronary Computed Tomography for Calcium Screening of Atherosclerosis
}

\author{
Joshua Beverly and Matthew J Budoff \\ Lundquist Institute at Harbor-UCLA Medical Center, Torrance, CA, USA
}

DOl: https://doi.org/10.17925/HI.2020.14.2.76

\begin{abstract}
$\mathrm{C}$ oronary artery calcium (CAC) scoring serves as a highly specific marker of coronary atherosclerosis. Based on the results of multiple large-scale, longitudinal population-based studies, CAC scoring has emerged as a reliable predictor of atherosclerotic cardiovascular disease (ASCVD) presence and risk assessment in asymptomatic patients across all age, sex and racial groups. Therefore, the measurement of CAC is useful in guiding clinical decision-making for primary prevention (e.g. use of statin and aspirin). This tool has already been incorporated into the clinical guidelines and is steadily being integrated into standard clinical practice. The adoption of CAC scoring will be important for curbing the progressive burden that ASCVD is exerting on our healthcare system. It has already been projected that CAC testing will decrease healthcare spending and will hopefully be shown to improve ASCVD outcomes. The purpose of this review is to summarise the evidence regarding calcium screening for atherosclerosis, particularly in asymptomatic individuals, including the pathophysiology, the prognostic power of CAC in the context of population-based studies, the progressive inclusion of CAC into clinical guidelines and the existing concerns of cost and radiation.
\end{abstract}

\section{Keywords}

Coronary artery calcium, atherosclerotic cardiovascular disease, statins, computed tomography scan, cardiovascular disease, primary prevention, atherosclerosis

Disclosures: Joshua Beverly and Matthew J Budoff have no financial or non-financial relationships or activities to declare in relation to this article.

Review Process: Double-blind peer review.

Compliance with Ethics: This study involves a review of the literature and did not involve any studies with human or animal subjects performed by any of the authors.

Authorship: The named authors meet the International Committee of Medical Journal Editors (ICMJE) criteria for authorship of this manuscript, take responsibility for the integrity of the work as a whole, and have given final approval for the version to be published.

Access: This article is freely accessible at

touchCARDIO.com (c) Touch Medical Media 2020.

Received: 6 November 2020

Accepted: 26 November 2020

Published Online: 17 December 2020

Citation: Heart International. 2020;14(2):76-9

Corresponding Author: Matthew Budoff, Lundquist Institute, 1124 W Carson Street, CDCRC, Torrance, CA 90502, USA. E: mbudoff@lundquist.org

Support: No funding was received in

the publication of this article.
Cardiovascular disease (CVD) represents the prevailing cause of death in the United States. Cancer, accidents and other prevalent causes of death, have a multitude of pathophysiologic and mechanistic underpinnings leading to mortality. Meanwhile, the majority of CVD is primarily attributable to atherosclerosis. ${ }^{2}$ Moreover, the degree of coronary calcification strongly correlates with the magnitude of atherosclerotic plaque burden, which is a strong and independent risk factor for coronary heart disease. ${ }^{3,4}$ Furthermore, the degree of coronary artery calcium (CAC) is predictive of atherosclerotic CVD (ASCVD) events, independent of patient demographics (e.g. age, race and gender). The traditional ASCVD risk calculators are imperfect, and CAC offers an additional level of refinement. Thus, measurement of coronary artery calcification by computed tomography (CT) scanning provides a low-cost, widely accessible modality to detect and guide treatment for persons with subclinical atherosclerosis.

The purpose of this review is to summarise the evidence regarding calcium screening for atherosclerosis, particularly in asymptomatic individuals, including the pathophysiology, the prognostic power of CAC in the context of population-based studies, the progressive inclusion of CAC into clinical guidelines, and the existing concerns of cost and radiation.

\section{Pathophysiology of coronary artery calcification}

Most individuals over age 60 have diffuse calcification throughout their vasculature. Previously, vascular calcification was thought to be a passive, degenerative consequence of aging. However, the development of vascular calcification is now recognized as a pathologic process based on ectopic bone formation, which is analogous to skeletal mineralisation. ${ }^{5}$ Atherogenesis begins with lipid retention in vulnerable arterial walls in the setting of endothelial dysfunction. ${ }^{6}$ Atherosclerotic plaques typically have a central necrotic core containing amorphous material bounded by a fibrous cap on the lumen side of the artery. Many parallels have been demonstrated between mineralisation of atherosclerotic plaques and bone, including the presence of type I collagen and crystalline hydroxyapatite facilitated by osteopontin, phosphatases and calcium binding phospholipids.' Lesions correlated with unstable angina or infarction are typically characterised by small calcium deposits described as 'spotty' or 'speckled', while stable angina is associated with a few, large, calcium deposits. ${ }^{8}$ It is important to note that the absence of coronary artery calcification on a particular segment does not necessarily preclude the presence of atherosclerosis in that segment. However, in the instances that calcification is minimal or absent, the lesion has typically only obstructed $0-25 \%$ of the lumen. ${ }^{3}$ For that reason, coronary CT stands as a powerful screening tool for coronary artery disease. 


\section{Coronary artery calcium scores in population-based cohort studies}

By convention, the most frequently used measure of CAC in the literature is the Agatston score. ${ }^{9}$ The protocol, developed by Dr Arthur Agatston, required an electron beam CT scanner without contrast to obtain $3 \mathrm{~mm}$ thick electrocardiogram gated to detect discrete calcific lesions defined as $>1 \mathrm{~mm}$ with a density $>130$ Hounsfield units. ${ }^{10}$ The calcium score was subsequently calculated based on the intensity of Hounsfield units and general age-based cutoffs were assigned; for example, 50 for patients in the 40-50 year age range and 300 for patients in their 60 s to predict clinical coronary artery disease. ${ }^{11}$ Since this investigation, which occurred in 1990, there have been multiple population-based cohort studies demonstrating the role of CAC in risk prediction - the largest one to date being the Multi-Ethnic Study of Atherosclerosis (MESA). ${ }^{11}$

The MESA trial is an epidemiologic, prospective cohort study designed to investigate the prevalence, risk factors, and progression of subclinical CVD in a multi-ethnic cohort that did not have any apparent clinical CVD. ${ }^{11}$ The study recruited 6,500 men and women aged 45-84 who self-identified as white, black, Hispanic, or Chinese from six different communities in the USA, from 2000 to $2002 .{ }^{12}$ Many measurements were obtained from the outset, including $\mathrm{CAC}$, values assessing traditional risk factors, cardiac magnetic resonance imaging, etc., in addition to psychosocial and socioeconomic factors, and participants were followed for at least 10 years. ${ }^{12}$ MESA demonstrated that there are differences in CAC score dependent on patient demographics beyond traditional risk factors. ${ }^{11}$ Nevertheless, multiple population-based studies have shown the reliability of CAC scores regardless of age, gender, or ethnicity, which upholds the utility of this strong subclinical marker. ${ }^{13}$

For practical purposes, the data from MESA has been used to formulate a predictive algorithm, known as the MESA risk calculator. The calculator was demonstrated to have improved performance, in comparison with the American College of Cardiology/American Heart Association (ACC/AHA) risk score, on two important properties of risk prediction models, discrimination and calibration. Discrimination refers to the ability of the model to differentiate those at higher risk of having an event from those at lower risk. ${ }^{14}$ For instance, the MESA risk score indicates that those who experience events will have 10-year risk estimates that are on average $8-9 \%$ higher than those with non-events. ${ }^{15}$ Calibration refers to the agreement between observed and predicted values in a given population. ${ }^{14}$ The risk score was externally validated outside of the development cohort in two other contemporary cohorts, the Dallas Heart Study and the Heinz Nixdorf Recall, with evidence of very good to excellent calibration. ${ }^{15}$ Inclusion of CAC scores into the MESA risk calculator provides significant accuracy in risk prediction.

CAC scores have low interscan variability (10\%), and as such, there are several studies longitudinally tracking CAC and associated outcomes. ${ }^{5}$ The progression of CAC seems to be most dependent on baseline CAC and ethnic background, and has minimal influence from traditional cardiovascular risk factors. ${ }^{16}$ The significance of CAC progression is that a temporal increase in CAC of $>15 \%$ is correlated with at least a 3.8-fold higher risk of first myocardial infarction if baseline CAC is $>100 .{ }^{17}$ This increased risk remained significant regardless of statin therapy and similar mean low-density lipids in both observational groups with and without statins. In regard to the best method to estimate risk, a study by Budoff et al. demonstrated in a cohort of 4,600 asymptomatic patients that employing the 'SQRT method', which is the difference between follow-up and baseline CAC score, was the best predictor of risk. ${ }^{18}$ This study also showed a significant increase in events for patients with CAC progression, except in patients with a baseline CAC of zero.

The value of discovering a patient with a CAC of zero cannot be understated. The utility of CAC was re-demonstrated in the Walter Reed Cohort study, which was a large-scale, observational study of 23,000 patients without baseline ASCVD, and differed from previous cohort studies in that the patients were generally younger (e.g. mean age was 50 years old compared with 62 years in MESA). In this study, there was a low mortality rate of $1 \%$ and major adverse cardiovascular event (MACE) rate of $2.7 \%{ }^{19} \mathrm{In}$ fact, the incidence rate of MACE and mortality was low enough to query whether there was any benefit of statin therapy in this subgroup of patients. A retrospective study performed on the Walter Reed Cohort sought to determine the magnitude of benefit provided by exposure to statin therapy across patient subgroups. The investigators found no benefit of statin therapy among patients without detectable CAC and classified as low or intermediate baseline ASCVD risk. ${ }^{20}$ Meanwhile, patients with a CAC of zero and high baseline ASCVD risk still demonstrated significant risk reduction with exposure to statin therapy. ${ }^{20}$ As it happens, Valentina et al. argue that no presence of CAC confers a '15-year warranty' against mortality regardless of age and gender, given the low annualized rate of mortality $(<1 \%)$ in their large-scale prospective study. ${ }^{21}$

In contrast, the same Walter Reed Cohort demonstrated the necessity of statin therapy in individuals with detectable CAC. CAC score severity was a significant predictor of ASCVD outcomes, independent of traditional risk factors. ${ }^{19}$ Practically speaking, statin therapy is more effective based on CAC severity as well, as evidenced by the aforementioned retrospective study. In the analysis, those with CAC $>100$ had the most significant risk reduction from statin therapy, with a 10-year number needed to treat of 12 to prevent one MACE. ${ }^{20}$ With the multitude of large-scale studies being published over the years in support of incorporating CAC scoring into risk prediction, it was only a matter of time before there would be a change on recommended clinical practice.

\section{Coronary artery calcium scores for clinical decision-making}

Classically, clinical practice guidelines have recommended risk assessment equations for quantitative estimation of absolute CVD risk. Important risk factors are based on office-based measurements, including age, total and high-density lipoprotein cholesterol, systolic blood pressure (treated or untreated status), diabetes and current smoking status. ${ }^{22}$ Unfortunately, it has been shown, in a large, contemporary and ethnically diverse population, that the 2013 ACC/AHA Pooled Cohort Risk Equations can substantially overestimate the ASCVD risk across sociodemographic subgroups. ${ }^{23}$ Resultantly, the overestimation of ASCVD risk can inadvertently lead to overtreatment of presumably significant risk factors, with the associated adverse effects and polypharmacy of such treatment. Calcium scoring via coronary CT can improve coronary risk estimation in order to avoid overtreatment and to provide assurance of low CVD risk when appropriate.

\section{Inclusion of coronary artery calcium into clinical guidelines}

The incorporation of CAC scoring into clinical practice guidelines has been gradual. The 2010 ACC/AHA guidelines on assessment of cardiovascular risk acknowledged the utility of CAC testing as a class $\mathrm{Ib}$ recommendation in individuals deemed 'intermediate risk' after formal risk assessment. ${ }^{24}$ More recently, the 2019 ACC/AHA 
guidelines on primary prevention provide more specified guidance on which individuals are most appropriate for CAC measurement. The guidelines listed CAC testing as a class Ila recommendation in intermediate-risk individuals, with a calculated ASCVD event risk of $7.5-20 \%$ by pooled cohort equations, as it can meaningfully reclassify a large proportion of individuals' need for statin therapy based on calcium score. ${ }^{25}$ For instance, potentially up to half of patients eligible for statin therapy, according to pooled cohort equations, but nevertheless, have a CAC of zero, have limited benefit from statins due to the low risk of ASCVD events in this population. ${ }^{26}$ Intermediate risk patients with a CAC of 1-99 have 10-year ASCVD event rates from 3.8-8.3\%, stratified across age. The current guidelines deem it reasonable to start statin therapy immediately or repeat risk assessment, including CAC, in 5 years. ${ }^{27}$ Moreover, a CAC $>100$ or $>75$ th age/sex/race percentile was consistently associated with an ASCVD event risk $>7.5 \%$; therefore, statin therapy would be indicated. Additional benefits of CAC measurement can be derived in 'borderline risk' individuals, especially if risk enhancers, such as family history of premature CVD, metabolic syndrome, chronic kidney disease or inflammatory diseases, are present. ${ }^{25}$ In regard to aspirin therapy, a subgroup analysis of MESA has shown that aspirin usage in patients with a CAC > 100 appears to have a favorable risk/benefit profile, while a CAC of zero shows a net harm. ${ }^{28}$

\section{Cost effectiveness of coronary calcium scoring}

The real-world practicality of CAC scoring will certainly be dependent on outcomes related to costs. In absolute terms, there is an increase in diagnostic costs and downstream testing across CAC strata, but the increase has been found to be appropriate by proportion, given the association of higher CAC scores with coronary artery disease severity and incidence of acute coronary syndrome. ${ }^{29}$ For instance, the diagnostic yield for obstructive coronary artery disease by invasive coronary angiography in the setting of acute chest pain was higher in patients with CAC $>400$ (87\%), compared with patients without CAC (25\%). ${ }^{29}$ Among patients with acute chest pain, an increasingly recognised phenomena that contributes to healthcare spending ischaemia with no obstructive coronary artery disease - is an emerging topic of investigation. ${ }^{30}$ It has been shown that $10 \%$ of all downstream tests, including invasive coronary angiography, were performed in men without CAC, none of which had acute coronary syndrome. ${ }^{29}$ This subgroup offers a possible target for change in practice and an opportunity to avoid unnecessary invasive testing. The same deduction cannot be expanded to women, as acute coronary syndrome occurred in $24 \%$ of women without CAC. Relatedly, elevated CAC was associated with greater risk of both 5-year mortality and MACE in symptomatic patients without significant luminal narrowing. ${ }^{31}$ Assuredly, future studies will be performed to delineate the role CAC plays, among other diagnostic modalities, for patients with ischaemia with no obstructive coronary artery disease.
Historically, there have been concerns regarding costs and radiation exposure associated with widespread screening, but investigation into these matters has found them of lesser consequence than initially suspected. Multiple simulations comparing treatment models based on traditional risk calculators versus risk estimation with inclusion of a calcium score have demonstrated that CAC testing is likely cost-saving when taking into account the decreasing costs of CAC testing, adverse effects, and actual cost of statin therapy, in addition to medication non-adherence and disutility. ${ }^{24,32}$ In fact, a systematic review and meta-analysis has suggested that identifying the presence of CAC has a twofold increase in the odds of initiation and continuation of aspirin, anti-hypertensives, anti-cholesterol medication, in addition to lifestyle interventions. ${ }^{33}$

Importantly, scanning for CAC does not inappropriately increase downstream medical resource utilisation and healthcare costs. ${ }^{34}$ The change in practice in response to the 2019 ACC/AHA guideline remains to be seen, given the recency of the updates. An obstacle in widespread adoption of CAC testing for screening is that the exam is not routinely covered by insurance companies, despite the relatively low cost of the scan - less than $\$ 200$. In contrast, there is much higher expenditure for colon and breast cancer screening; namely, reimbursements from insurance companies for tests that cost over $\$ 3,000 .{ }^{2}$ The unwillingness to cover this powerful prevention measure - CAC testing - is not sensible in light of the healthcare costs spent on ASCVD-related morbidity and mortality. An overstated risk of routine screening with CAC is the exposure to radiation. Due to developing reconstructive algorithms, a CAC scan is only $1 \mathrm{mSv}$, which is almost equivalent to screening mammography, and is likely inconsequential according to the American Association of Physicists in Medicine. ${ }^{35}$ In short, CAC testing is low-cost with minimal exposure to radiation and, therefore, a perfect tool for widespread screening efforts for the reduction of ASCVD events.

\section{Conclusion}

CVD is an ever-present reality in developed and developing countries, and its accompanying morbidity and mortality is bound to have an increasing impact on society. Additionally, the associated healthcare costs to address this worsening situation will certainly take a toll, worldwide. In response, our approaches need to be more directed and more tactical. CAC testing offers that opportunity. In the future, investigation into improvement of ASCVD outcomes from the incorporation of CAC scoring would lend further credence that this modality should be standardised, in the hope that providers can elect to order CAC scanning to the benefit of the patient without penalty from insurance companies. In addition to risk-factor-based paradigms, CAC measurement affords another layer of precision for preventative management in our evolving world. $\square$
1. Virani SS, Alonso A, Benjamin $\mathrm{EJ}$, et al. On behalf of the American Heart Association Council on Epidemiology and Prevention Statistics Committee and Stroke Statistics and Prevention Statistics Committee and Stroke Statistics Subcommittee. Heart Disease and Stroke Statistics-2020
Update: A Report From the American Heart Association.

2. Naghavi M, Maron DJ, Kloner RA, et al. Coronary artery calcium testing: A call for universal coverage. Prev Med Rep. 2019;15:100879.

3. Sangiorgi G, Rumberger JA, Severson A, et al. Arterial calcification and not lumen stenosis is highly correlated with atherosclerotic plaque burden in humans: A histologic study of 723 coronary artery segments using nondecalcifying methodology. J Am Coll Cardiol. 1998;31:126-33.

4. Allison MA, Criqui MH, Wright CM. Patterns and risk factors for systemic calcified atherosclerosis. Arterioscler Thromb vasc Biol. 2004:24:331-6.

5. Greenland P, Blaha MJ, Budoff MJ, et al. Coronary calcium score and cardiovascular risk. J Am Coll Cardio. 2018;72:434-47.
6. Beverly JK, Budoff MJ. Atherosclerosis: Pathophysiology of insulin resistance, hyperglycemia, hyperlipidemia, and inflammation. J Diabetes. 2019:12:102-4.

7. Fitzpatrick LA, Severson A, Edwards WD, Ingram RT. Diffuse Fitzpatrick $L A$, Severson $A$, Edwards WD, Ingram RT. Diffuse
calcification in human coronary arteries. Association of osteopontin with atherosclerosis. J Clin Invest. 1994;94:1597-604

8. Demer LL, Tintut $Y$. Vascular calcification: pathobiology of a multifaceted disease. Circulation. 2008;117:2938-48.

9. Agatston AS, Janowitz WR, Hildner FJ, et al. Quantification of coronary artery calcium using ultrafast computed tomography. J Am Coll Cardiol. 1990;15:827-32

10. Thompson GR, Forbat S, Underwood R. Electron-beam CT scanning for detection of coronary calcification and prediction of coronary heart disease. QJM. 1996;89:565-70.

11. McClelland RL, Chung $H$, Detrano $R$, et al. Distribution of coronary artery calcium by race, gender, and age: Results from the Multi-Ethnic Study of Atherosclerosis (MESA). Circulation. 2006:113:30-7.

12. Bild DE. Multi-ethnic study of atherosclerosis: Objectives and design. Am J Epidemiol. 2002:156:871-81.

13. Shaikh K, Nakanishi R, Kim N, Budoff MJ. Coronary artery calcification and ethnicity. $J$ Cardiovasc Comput Tomog. 2019;13:353-9.

14. Alba AC, Agoritsas T, Walsh M, et al. Discrimination and calibration of clinical prediction models: Users' guides to the medical literature. JAMA. 2017;318:1377-84.

15. McClelland RL, Jorgensen NW, Budoff M, et al. Ten-year coronary heart disease risk prediction using coronary artery calcium and traditional risk factors: derivation in the multi-ethnic study of atherosclerosis with validation in the Heinz Nixdorf Recall Study and the Dallas Heart Study. J Am Coll Cardiol. 2015;66:1643-53.

16. Erbel R, Lehmann N, Churzidse S, et al. Progression of coronary artery calcification seems to be inevitable, but predictableresults of the Heinz Nixdorf Recall (HNR) study. Eur Heart J. 2014:35:2960-71.

17. Raggi $P$, Callister TQ, Shaw $L$ J. Progression of coronary artery calcium and risk of first myocardial infarction in patients 
receiving cholesterol-lowering therapy. Arterioscler Thromb Vasc Biol. 2004;24:1272-7.

18. Budoff MJ, Hokanson JE, Nasir K, et al. Progression of coronary artery calcium predicts all-cause mortality. JACC CardiovasC 229-36.

19. Mitchell JD, Paisley R, Moon P, et al. Coronary artery calcium and long-term risk of death, myocardial infarction, and stroke: The Walter Reed Cohort Study. JACC Cardiovasc Imaging. 2018;11:1799-806

20. Mitchell JD, Fergestrom N, Gage BF, et al. Impact of statins on cardiovascular outcomes following coronary artery calcium scoring. J Am Coll Cardiol. 2018;72:3233-42.

21. Valentina V, Hartaigh BÓ, Gransar H, et al. A 15-year warranty period for asymptomatic individuals without coronary artery calcium: A prospective follow-up of 9,715 individuals JACC CardiovasC Imaging. 2015;8:900-9.

22. Goff DC, Lloyd-Jones DM, Bennett G, et al. 2013 ACC/AHA guideline on the assessment of cardiovascular risk: A report of the American College of Cardiology/American Heart Association Task Force on Practice Guidelines. A Am Coll Cardiol. 2014;63:2935-59.

23. Rana JS, Tabada GH, Solomon MD, et al. Accuracy of the atherosclerotic cardiovascular risk equation in a large contemporary, multiethnic population. J Am Coll Cardiol. 2016;67:2118-30.

24. Greenland P, Alpert JS, Beller GA, et al. 2010 ACCF/AHA guideline for assessment of cardiovascular risk in asymptomatic adults: Executive summary: A report of the American College of Force on Practice Guidelines. Circulation. 2010;122: Force on $2748-64$.

25. Arnett DK, Blumenthal RS, Albert MA, et al. 2019 ACC/AHA guideline on the primary prevention of cardiovascular disease: A Report of the American College of Cardiology/American Heart Association Task Force on Clinical Practice Guidelines. Circulation. 2019;140:e596-e646.

26. Nasir K, Bittencourt MS, Blaha MJ, et al. Implications of coronary artery calcium testing among statin candidates according to American College of Cardiology/American Heart Association Cholesterol Management Guidelines: MESA (Multi-Ethnic Study of Atherosclerosis. J Am Coll Cardiol. 2015;66:1657-68.

27. Budoff MJ, Young R, Burke G, et al. Ten-year association of coronary artery calcium with atherosclerotic cardiovascular disease (ASCVD) events: The multi-ethnic study of atherosclerosis (MESA). Eur Heart J. 2018;39:2401-8.

28. Miedema MD, Duprez DA, Misialek JR, et al. The use of coronary artery calcium testing to guide aspirin utilization coronary artery calcium testing to guide aspirin utilization
for primary prevention: Estimates from the Multi-Ethnic Study of Atherosclerosis. Circ Cardiovasc Qual Outcomes Study of Atherosc

29. Bittner DO, Mayrhofer T, Bamberg F, et al. Impact of coronary calcification on clinical management in patients with acute chest pain. Circ Cardiovasc Imaging. 2017;10:e005893.

30. Herscovici R, Sedlak T, Wei J, et al. Ischemia and no obstructive coronary artery disease (INOCA): What is the risk? I Am Heart Assoc. 2018:7:e008868.

31. Petretta $M$, Daniele $S$, Acampa W et al Prognostic value of coronary artery calcium score and coronary CT angiography in patients with intermediate risk of coronary artery disease. Int J Cardiovasc Imaging. 2012,28.1547-56.

32. Roberts ET, Horne A, Martin SS, et al. Cost-effectiveness of coronary artery calcium testing for coronary heart and cardiovascular disease risk prediction to guide statin allocation: The Multi-Ethnic Study of Atherosclerosis (MESA). PLOS One. 2015;10:e0116377.

33. Gupta A, Lau E, Varshney R, et al. The identification of calcified coronary plaque is associated with initiation and continuation of pharmacological and lifestyle preventive therapies: A systematic review and meta-analysis. JACC Cardiovas Imaging. 2017;10:833-42.

34. Rozanski A, Gransar H, Shaw $\sqcup$, et al. Impact of coronary artery calcium scanning on coronary risk factors and downstream calcium scanning on coronary risk factors and downs
testing the EISNER (Early Identification of Subclinical testing the EISNER (Early Identification of Subclinical
Atherosclerosis by Noninvasive Imaging Research) prospective Atherosclerosis by Noninvasive Imaging Research) prosp

randomized trial. J Am Coll Cardiol. 2011;57:1622-32.
35. Hecht HS, Henschke C, Yankelevitz D, et al. Combined detection of coronary artery disease and lung cancer. Eur Heart $\mathrm{J}$. 2014;35:2792-6. 